\title{
Clinical Study \\ Stereotactic Body Radiotherapy for Metastatic Lung Cancer as Oligo-Recurrence: An Analysis of 42 Cases
}

\author{
Wataru Takahashi, ${ }^{1}$ Hideomi Yamashita, ${ }^{1}$ Yuzuru Niibe, ${ }^{2}$ Kenshiro Shiraishi, ${ }^{1}$ \\ Kazushige Hayakawa, ${ }^{2}$ and Keiichi Nakagawa ${ }^{1}$ \\ ${ }^{1}$ Department of Radiology, University of Tokyo Hospital, 7-3-1, Hongo, Bunkyo-ku, Tokyo 113-8655, Japan \\ ${ }^{2}$ Department of Radiology and Radiation Oncology, Kitasato Universtiy, Kanagawa 252-0374, Japan \\ Correspondence should be addressed to Hideomi Yamashita, yamachan07291973@yahoo.co.jp
}

Received 11 June 2012; Revised 3 August 2012; Accepted 3 September 2012

Academic Editor: Takao Hiraki

Copyright (C) 2012 Wataru Takahashi et al. This is an open access article distributed under the Creative Commons Attribution License, which permits unrestricted use, distribution, and reproduction in any medium, provided the original work is properly cited.

\begin{abstract}
Purpose. To investigate the outcome and toxicity of stereotactic body radiotherapy (SBRT) in patients with oligo-recurrence cancer in the lung (ORCL). Methods and Materials. A retrospective review of 42 patients with ORCL who underwent SBRT in our two hospitals was conducted. We evaluated the outcome and adverse effects after SBRT for ORCL. Results. All patients finished their SBRT course without interruptions of toxicity reasons. The median follow-up period was 20 months (range, 1-90 months). The 2 -year local control rate and overall survival were 87\% (95\% CI, 75-99\%) and 65\% (95\% CI, 48-82\%). As for prognostic factor, the OS of patients with a short disease-free interval (DFI) $<31.9$ months, between the initial therapy and SBRT for ORCL, was significantly worse than the OS of long DFI $\geqq 31.9$ months $(P<0.05)$. The most commonly observed late effect was radiation pneumonitis. One patient had grade 4 gastrointestinal toxicity (perforation of gastric tube). No other $\geqq$ grade 3 acute and late adverse events occurred. There were no treatment-related deaths during this study. Conclusions. In patients with ORCL, radical treatment with SBRT is safe and provides a chance for long-term survival by offering favorable local control.
\end{abstract}

\section{Introduction}

Lung is one of the common sites of metastasis after definitive therapy for a primary cancer. So far, recurrent or metastatic lung cancers have been considered to uniformly carry a poor prognosis because multiple metastases tend to be difficult to treat intensively. Chemotherapy has been broadly applied as a standard management at these conditions. On the other hand, the innovation of methods of early detection of recurrence, such as positron-emission tomography (PET), allows the detection of limited site recurrent, called oligorecurrence. Oligo-recurrence, proposed by Niibe et al. in 2006 [1-4], was the condition of one or a few metastatic or recurrent lesions occurred with controlled primary lesion. For case with oligo-recurrence cancer in the lung (ORCL), the controversy exists regarding the optimal approach of these metastatic sites. Despite surgical approach is considered as an alternative for a single metastasis, there are many patients with ORCL who were not amenable for metastasectomy. For them, less invasive techniques such as SBRT have been used to treat ORCL. In cases considered to have a favorable prognosis, radical treatment with SBRT seems to be beneficial for prolonging the survival time. However, the role of radiotherapy and the prognostic factors for oligorecurrence have not yet been clearly elucidated [5]. In this study, we evaluated the efficacy and toxicity of SBRT for patients with oligo-recurrence cancer treated from 2001 through 2011 in two hospitals.

\section{Materials and Methods}

2.1. Patient Eligibility and Pretreatment Evaluation. A retrospective review of all patients with ORCL treated with SBRT after prior therapy at University of Tokyo Hospital and Kitasato University Hospital from April 2001 to July 2011 was conducted. Patients with ORCL who were not suitable for surgery due to medical or functional reasons were included in this analysis. Pretreatment evaluation included a complete medical history, physical examination, computed tomography $(\mathrm{CT})$, pulmonary function tests, and laboratory tests. In 
addition, 36 of 42 patients (86\%) were evaluated with ${ }^{18} \mathrm{~F}$ fluorodeoxyglucose (FDG)-PET before treatment. Inclusion criteria of this study were as follows: (a) primary cancer was completely treated; (b) the number of lung metastases were up to three; (c) there was no other distant metastasis or other distant metastasis was scheduled to be treated with curative intent after SBRT. As long as these evaluations fulfilled the inclusion criteria, there was no restriction regarding tumor size, location, or general pulmonary function. Radiotherapy was the exclusive treatment modality in all patients.

2.2. Radiotherapy. SBRT was given with $6 \mathrm{MV} \mathrm{X}$-ray of a linear accelerator. In curative intention, hypofractionated SBRT was delivered to a median dose of 48 Gy (range, 20$56 \mathrm{~Gy}$ ) with a median daily dose of $12 \mathrm{~Gy}$ (range, 8-30 Gy). Dose and fractionation schedules were chosen depending on location and institution. In University of Tokyo Hospital, SBRT was performed using the Synergy linear accelerator (ELEKTA), which fully integrates IGRT by means of $\mathrm{kV}$-CT scanning. In Kitasato University Hospital, real-time tumortracking radiotherapy was used for SBRT. The gross tumor volume (GTV) or internal target volume (ITV) included the visible gross tumor mass on CT were delineated on a threedimensional radiation treatment planning system (3D RTPS) using the lung window. The planning target volume (PTV) was created by adding five mm margin to the ITVs in all directions.

2.3. Follow-Up. After completion of therapy, patients were scheduled for regular follow-up visits 3 monthly during the first year, 6 monthly thereafter. Those who did not appear for a routine follow-up were contacted by phone. Follow-up evaluations included a history and physical examination and CT scans of the thorax. Additional imaging investigations such as FDG-PET were only required if there was clinical suspicion of recurrence. In this study, we define local recurrence as an increase in opacity size on CT imaging, along with either increased maximum standardized uptake values (SUVmax) $\geqq 5$ on FDG-PET, or biopsy proof of disease [6]. Toxicity was evaluated and scored according to the National Cancer Institute Common Terminology Criteria for Adverse Events (NCI CTCAE) version 4.0, with toxicity occurring within 3 months after the initiation of RT classified as acute toxicity. Late toxicity was graded using the RTOG/EORTC criteria.

2.4. Statistical Analysis. The baseline follow-up date was the first day of radiotherapy, and the last follow-up date was the last Hospital visit or phone day. Overall survival (OS) was calculated from the start of the SBRT to the date of death, censoring the last follow-up date. Local control rate (LCR) was calculated from the start of the SBRT to the first local recurrence date, censoring death or last follow-up date.

To discuss risk factors for OS and LCR, the patients of ORCL were classified into two groups: early recurrence group and late recurrence group. The former group consisted of 21 patients whose disease-free interval (DFI), meaning interval between the start date of initial therapy and the start date of SBRT for ORCL, was shorter than 31.9 months (median
DFI time). In addition, we compared OS and LCR following SBRT for ORCL from colorectal cancer (CRC) and other origins. OS and LCR curves were plotted using the KaplanMeier method. Log-rank testing was used to compare OS and LCR between the subsets of patients analyzed. All analyses were performed using SPSS software version 12.0 (SPSS Inc., Chicago, IL).

\section{Results}

From April 2001 to July 2011, we identified 42 patients with ORCL who were treated with SBRT. The median age was 69 years (range, 25-84 years). There were 30 men and 12 women. The median maximum diameter of metastatic tumor was $19 \mathrm{~mm}$ (range, 9-40 mm). Patient characteristics are shown in Table 1. One patient underwent chemotherapy for ORCL before SBRT and the other 41 patients did not undergo neoadjuvant, concurrent, or adjuvant chemotherapy for ORCL. Sites of primary disease included lung $(n=$ 16), colon and rectum $(n=7)$, head and neck (6), esophagus $(n=4)$, uterus $(n=4)$, kidney $(n=2)$, and others (renal pelvis, breast, sarcoma; $n=3$ ). Of these, 32 patients had lung metastasis alone, 8 patients had another lung metastasis treated with SBRT after initial SBRT, and 2 patients had a distant metastasis in addition to lung lesion (retroperitoneal node and adrenal gland). These distant metastases in both patients were also treated with SBRT after completing SBRT for lung lesion. At the time to analysis, they were alive without evidence of any recurrence.

All patients finished their SBRT course without interruptions of toxicity reasons. Acute toxicities were mild and tolerable except for one case. Grade 4 acute adverse event were observed in only 1 patient (2\%), which displayed the perforation of the pulled-up gastric tube. This patient was a 59-year-old man, with esophageal cancer after total esophagectomy with esohageal replacement by means of a gastric tube, had undergone SBRT, consisting of $50 \mathrm{~Gy}$ in four fractions in 4 days. The $\mathrm{D} 2 \mathrm{cc}$, the minimum dose in the most irradiated $2 \mathrm{cc}$ of the gastric tube, was $48.66 \mathrm{~Gy}$. He was a heavy smoker and had an alcohol problem. Two months later, he developed perforation of the gastric tube.

No other grade $\geq 3$ acute side effects occurred. Twentyone patients (50\%) and 5 patients (12\%) experienced grade 1 and 2 adverse event after irradiation of metastases, respectively. Of the 42 patients, 21 patients $(50 \%)$ and 3 patients (7\%) displayed grade 1 pneumonitis (asymptomatic, radiographic findings only) and grade 2 pneumonitis (symptomatic, not interfering with activities of daily living), respectively. No grade $\geq 3$ late adverse events occurred until now. The median duration of follow-up was 20 months (range, 1-90 months) for all patients and 24 months (range, 6-90 months) for those alive. The 1- and 2-year local control rates were 91\% (95\% CI, 82-100\%) and 87\% (95\% CI, 75$99 \%$ ), respectively (Figure 1). At the time of last follow-up, 16 patients had died. The causes of death were recurrence $(n$ $=9)$, other diseases $(n=7)$. The overall 1 - and 2-year survival rates were $81 \%(95 \% \mathrm{CI}, 69-94 \%)$ and $65 \%$ (95\% CI, $48-$ $82 \%$ ), respectively (Figure 1), with a median survival time 
TABle 1: Patients characteristics $(n=42)$.

\begin{tabular}{|c|c|c|c|}
\hline Variable & Distribution & No. of patients & $\%$ \\
\hline \multirow{2}{*}{ Sex } & Male & 30 & 71 \\
\hline & Female & 12 & 29 \\
\hline \multirow{2}{*}{ Age } & Median & 69 years & \\
\hline & Range & $25-84$ years & \\
\hline \multirow{2}{*}{ Karnofsky Performance status } & Median & 90 & \\
\hline & Range & $50-90$ & \\
\hline \multirow{3}{*}{ Number of metastases } & 1 & 32 & 76 \\
\hline & 2 & 10 & 24 \\
\hline & $\geqq 3$ & 0 & 0 \\
\hline \multirow{2}{*}{ Maximum diameter (mm) } & Median & $19 \mathrm{~mm}$ & \\
\hline & Range & $9-40 \mathrm{~mm}$ & \\
\hline \multirow{7}{*}{ Primary site } & Lung & 16 & 38 \\
\hline & Colon and rectum & 7 & 17 \\
\hline & Head and neck & 6 & 14 \\
\hline & Esophagus & 4 & 10 \\
\hline & Uterus & 4 & 10 \\
\hline & Kidney & 2 & 5 \\
\hline & Other & 3 & 5 \\
\hline \multirow{2}{*}{ Follow-up (months) } & Median & 20 months & \\
\hline & Range & $1-90$ months & \\
\hline
\end{tabular}

of 40 months. Seventeen of 42 patients showed a long-term survival of longer than 2 years.

In present study, seven patients with ORCL originated from CRC and 35 patients originated from other origins were treated by SBRT. The 1- and 2-year LCR in ORCL from CRC and in ORCL from other origins were $83 \%$ and $67 \%$, $89 \%$ and $89 \%$, respectively (Figure 2 ). The overall $1-$ and 2year survival rates in ORCL from CRC and in ORCL from other origins were $85 \%$ and $85 \%, 82 \%$ and $63 \%$, respectively (Figure 3$)$. These results showed no significant difference in $\operatorname{LCR}(P=0.31)$ and $\mathrm{OS}(P=0.26)$.

We also analyzed the LCR and OS differences stratified by DFI divided into $<31.9$ or $\geqq 31.9$ months. As shown in Figure 4, the result indicated a negative correlation between DFI and LCR $(P=0.29)$. On the other hand, early recurrence group (short DFI) had significantly bad prognosis $(P<0.05$; Figure 5).

\section{Discussion}

Although this is a retrospective study with a limited sample size, our results are also comparable to other studies in ORCL [7-9]. Norihisa et al. [10] also previously showed the results of SBRT for 43 metastatic lung cancers. In their series, the survival rates and local control rate at 2 years were reported to be $84.3 \%$ and $90 \%$, respectively. Ricardi et al. [11] also reported a study of SBRT for oligometastatic lung tumors. Sixty-one patients treated with SBRT achieved $89 \%$ in local control and $66.5 \%$ in survival at 2 years.
Several studies have now shown that the local control after SBRT for lung metastases from CRC is worse than that from other origins. Takeda et al. [12] reported the difficulty of local control for ORCL from CRC. Norihisaet al. [10] proposed dose escalation in SBRT for CRC patients in order to achieve better local control. In the current study, there was no significant difference between CRC and other origins in LCR $(P=0.31)$ and OS $(P=0.26)$, respectively.

Furthermore, we also analyzed the OS and LCR differences stratified by DFI divided into $<31.9$ or $\geqq 31.9$ months. As shown in Figure 5, short DFI was the prognostic factor $(P$ $<0.05)$. Thus, even as oligo-recurrence, early metastasis may be bad prognostic factor.

It seems from these results that SBRT is an effective and safe treatment for patients with lung metastases as oligorecurrence. In SBRT for lung metastases, limited toxicity rates are reported by several authors [12]. In our series, there was no patient with serious late toxicities except for one patient with perforation of gastric tube. Although it is likely that the perforation may be caused mainly by radiation to gastric tube, smoking and bad nutrition might have been partly related to this perforation. Several reports advocated that deterioration in smoking and bad nutritional status during radiotherapy could be associated with poorer shortterm treatment outcomes and severe side effect $[13,14]$.

Several limitations of this study warrant mention. First, it was a retrospective review with a limited number of patients and limited follow-up. Second, we treated ORCL from various primary cancers by using different treatment 


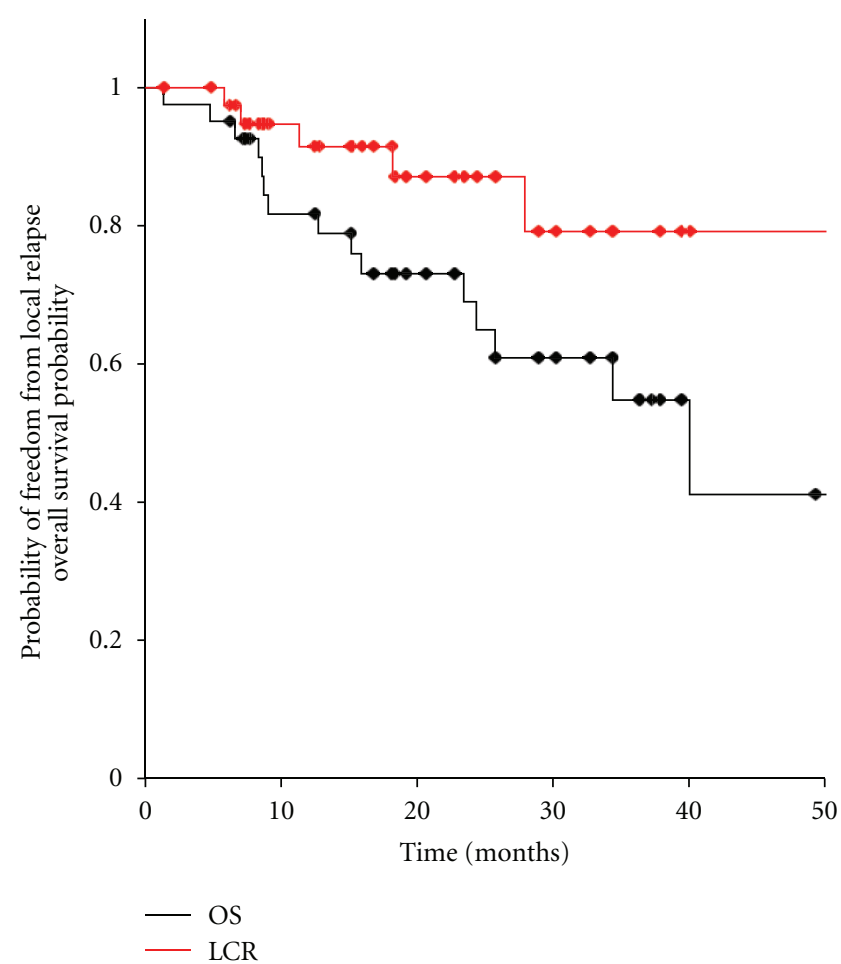

Figure 1: Overall survival and local control of 42 patients with oligo-recurrence cancer in the lung.

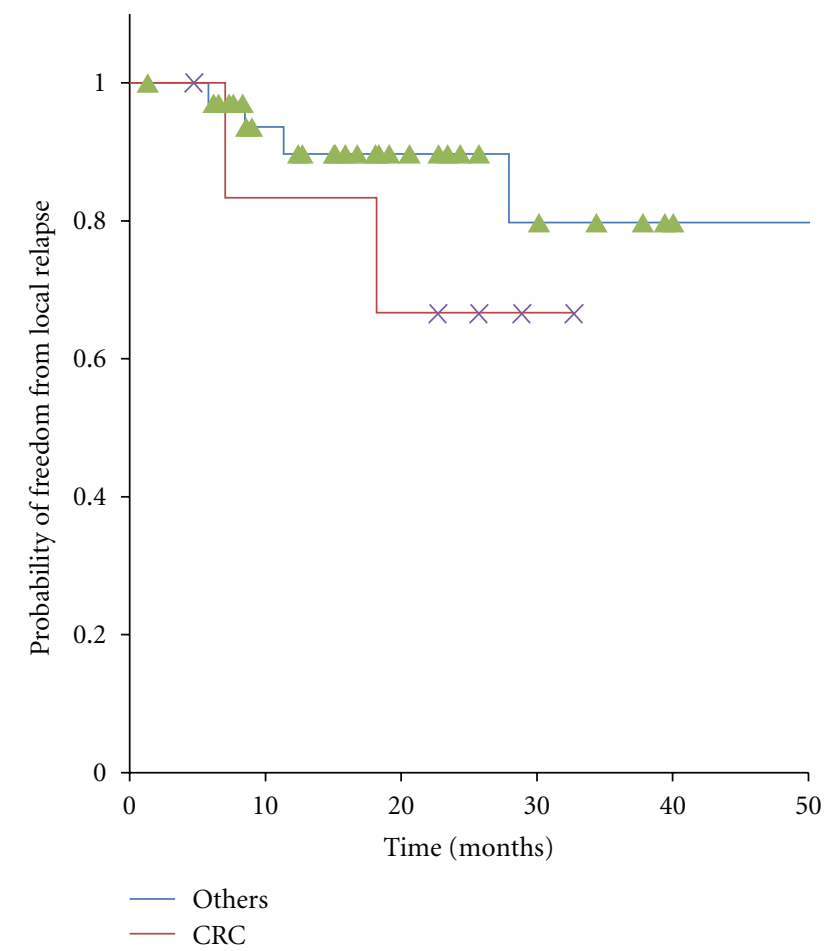

Figure 2: Kaplan-Meier curves for local control in 42 patients with oligo-recurrence cancer in the lung, cancers from colorectal cancer and ones from other origins.

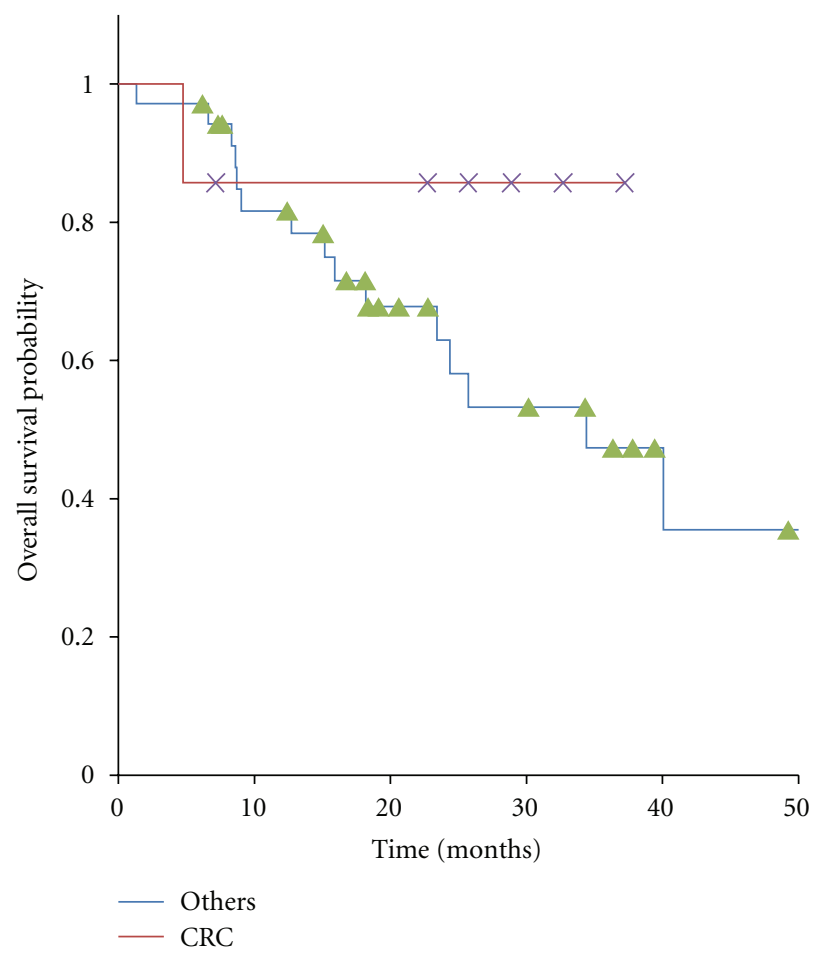

Figure 3: Kaplan-Meier curves for overall survival in 42 patients with oligo-recurrence cancer in the lung, cancers from colorectal cancer and ones from other origins.

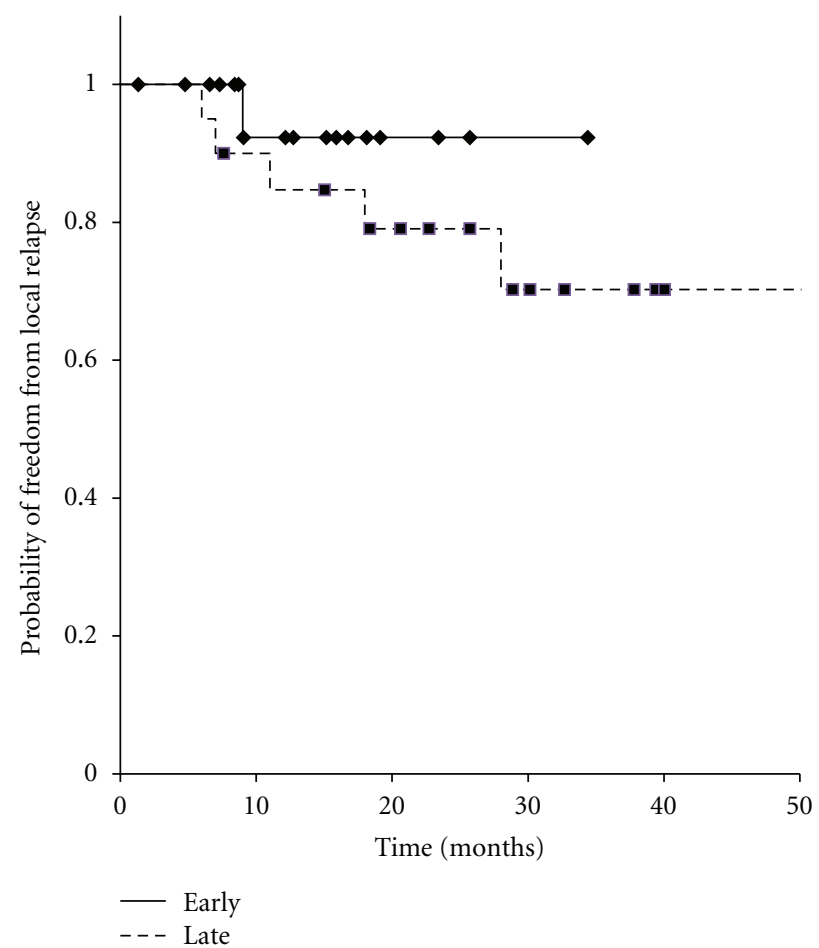

FIGURE 4: Kaplan-Meier curves for local control in 42 patients with oligo-recurrence cancer in the lung, early recurrence group versus late recurrence group. 


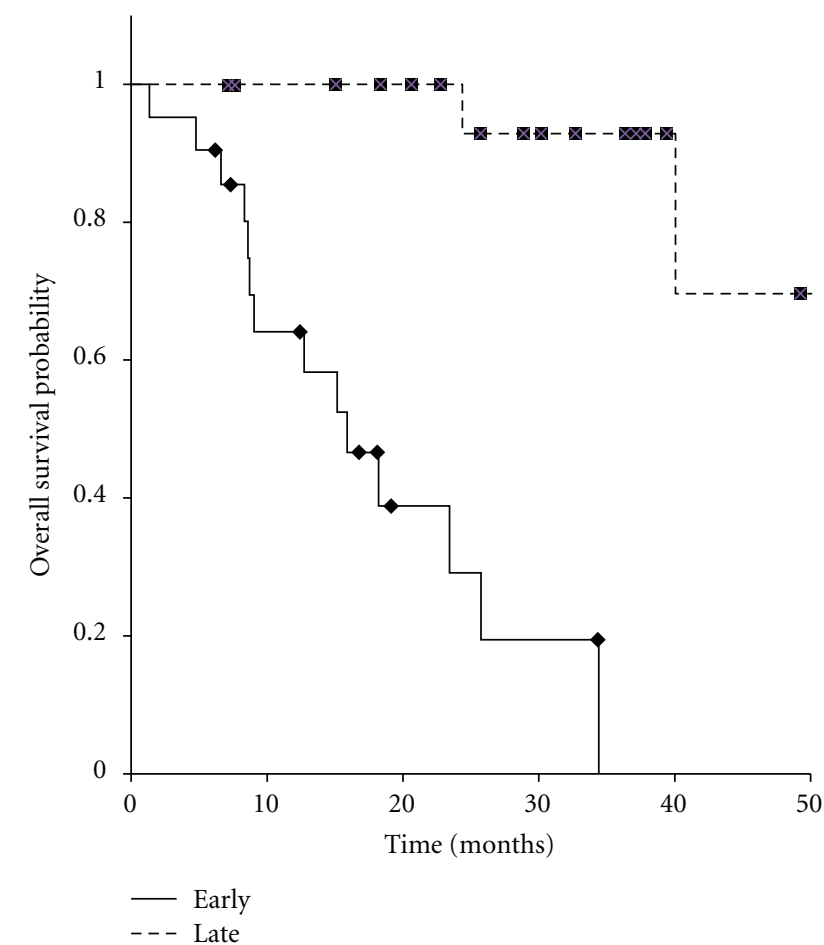

Figure 5: Kaplan-Meier curves for overall survival in 42 patients with oligo-recurrence cancer in the lung, early recurrence group versus late recurrence group.

protocol. There was a wide range of doses prescribed, and a variety of fractionation schema.

\section{Conclusions}

In patients with ORCL, radical treatment with SBRT offers good local control and provides a real chance for long-term survival. In addition, even in ORCL, SBRT is a safe and efficacious modality and appears to be well tolerated.

\section{Conflict of Interests}

The authors declare that they have no conflict of intrersts.

\section{References}

[1] Y. Niibe, T. Kazumoto, T. Toita et al., "Frequency and characteristics of isolated para-aortic lymph node recurrence in patients with uterine cervical carcinoma in Japan: a multiinstitutional study," Gynecologic Oncology, vol. 103, no. 2, pp. 435-438, 2006.

[2] Y. Niibe, M. Kenjo, T. Kazumoto et al., "Multi-institutional study of radiation therapy for isolated para-aortic lymph node recurrence in uterine cervical carcinoma: 84 subjects of a population of more than 5,000," International Journal of Radiation Oncology Biology Physics, vol. 66, no. 5, pp. 13661369, 2006.

[3] Y. Niibe, M. Kuranami, K. Matsunaga et al., "Value of highdose radiation therapy for isolated osseous metastasis in breast cancer in terms of oligo-recurrence," Anticancer Research, vol. 28, no. 6, pp. 3929-3931, 2008.

[4] Y. Niibe and K. Hayakawa, "Oligometastases and oligorecurrence: the new era of cancer therapy," Japanese Journal of Clinical Oncology, vol. 40, no. 2, pp. 107-111, 2010.

[5] S. K. Jabbour, P. Daroui, D. Moore, E. Licitra, M. Gabel, and J. Aisner, "A novel paradigm in the treatment of oligometastatic non-small cell lung cancer," Journal of Thoracic Disease, vol. 3 , no. 1, pp. 4-9, 2011.

[6] K. Huang, M. Dahele, S. Senan et al., "Radiographic changes after lung stereotactic ablative radiotherapy (SABR) — can we distinguish recurrence from fibrosis? A systematic review of the literature," Radiotherapy \& Oncology, vol. 102, no. 3, pp. 335-342, 2012.

[7] P. Okunieff, A. L. Petersen, A. Philip et al., "Stereotactic body radiation therapy (SBRT) for lung metastases," Acta Oncologica, vol. 45, no. 7, pp. 808-817, 2006.

[8] K. E. Rusthoven, B. D. Kavanagh, S. H. Burri et al., "Multiinstitutional phase I/II trial of stereotactic body radiation therapy for lung metastases," Journal of Clinical Oncology, vol. 27, no. 10, pp. 1579-1584, 2009.

[9] D. Oh, Y. C. Ahn, J. M. Seo et al., "Potentially curative stereotactic body radiation therapy (SBRT) for single or oligometastasis to the lung," Acta Oncologica, vol. 51, no. 5, pp. 596-602, 2012.

[10] Y. Norihisa, Y. Nagata, K. Takayama et al., "Stereotactic body radiotherapy for oligometastatic lung tumors," International Journal of Radiation Oncology Biology Physics, vol. 72, no. 2, pp. 398-403, 2008.

[11] U. Ricardi, A. R. Filippi, A. Guarneri et al., "Stereotactic body radiation therapy for lung metastases," Lung Cancer, vol. 75, no. 1, pp. 77-81, 2012.

[12] A. Takeda, E. Kunieda, T. Ohashi, Y. Aoki, N. Koike, and T. Takeda, "Stereotactic body radiotherapy (SBRT) for oligometastatic lung tumors from colorectal cancer and other primary cancers in comparison with primary lung cancer," Radiotherapy \& Oncology, vol. 101, no. 2, pp. 255-259, 2011.

[13] A. Hill, N. Kiss, B. Hodgson, T. C. Crowe, and A. D. Walsh, "Associations between nutritional status, weight loss, radiotherapy treatment toxicity and treatment outcomes in gastrointestinal cancer patients," Clinical Nutrition, vol. 30, no. 1, pp. 92-98, 2011.

[14] C. M. Hoff, C. Grau, and J. Overgaard, "Effect of smoking on oxygen delivery and outcome in patients treated with radiotherapy for head and neck squamous cell carcinoma-a prospective study," Radiotherapy \& Oncology, vol. 103, no. 1, pp. 38-44, 2012. 


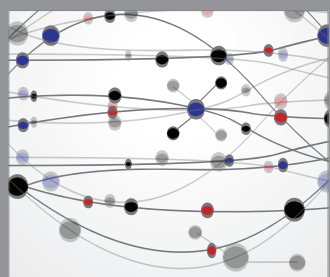

The Scientific World Journal
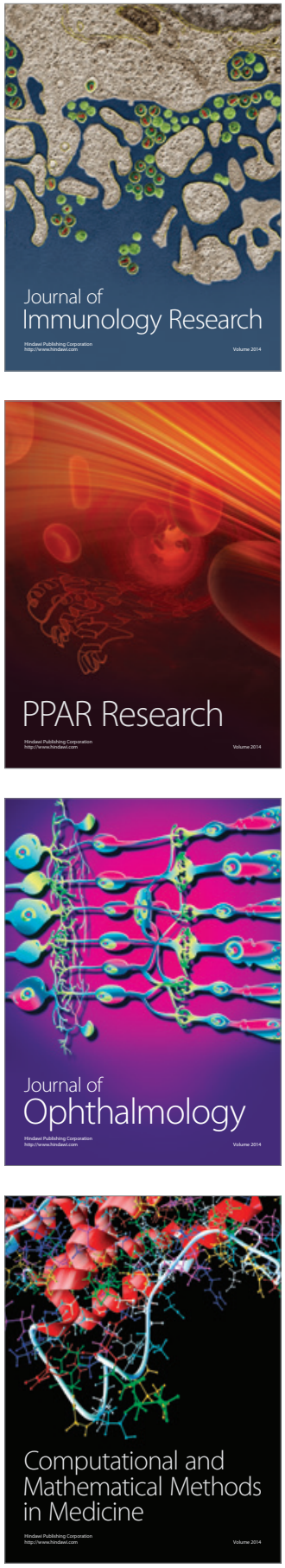

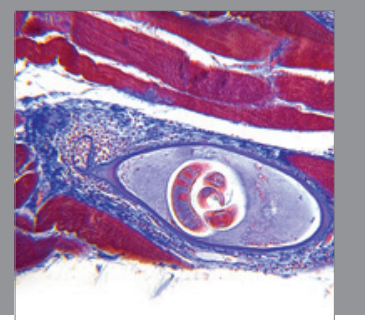

Gastroenterology

Research and Practice
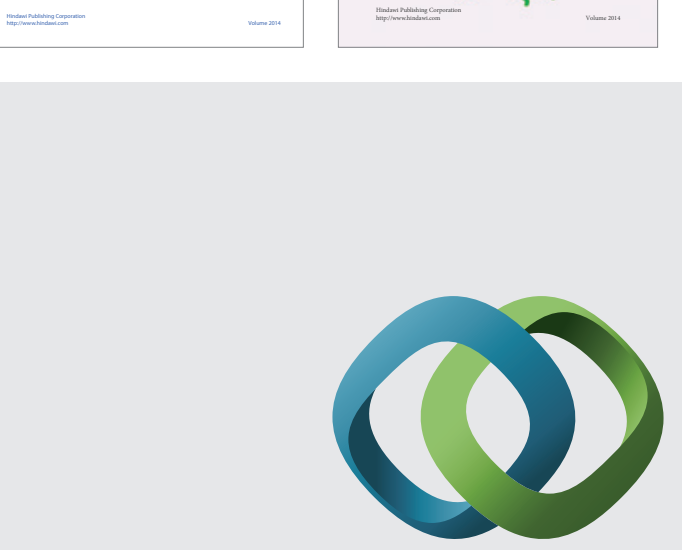

\section{Hindawi}

Submit your manuscripts at

http://www.hindawi.com
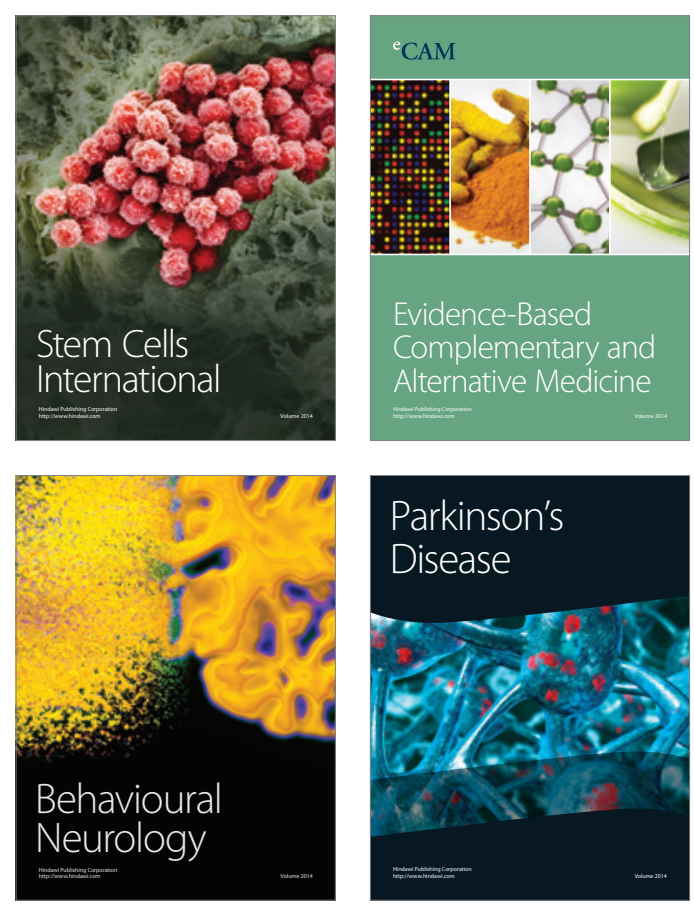

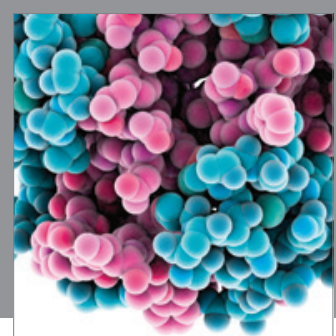

Journal of
Diabetes Research

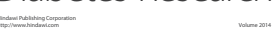

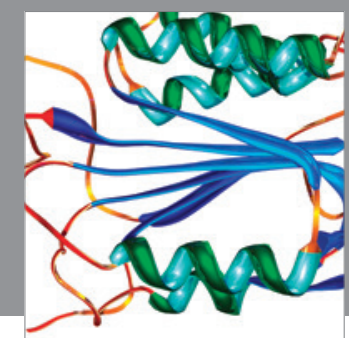

Disease Markers
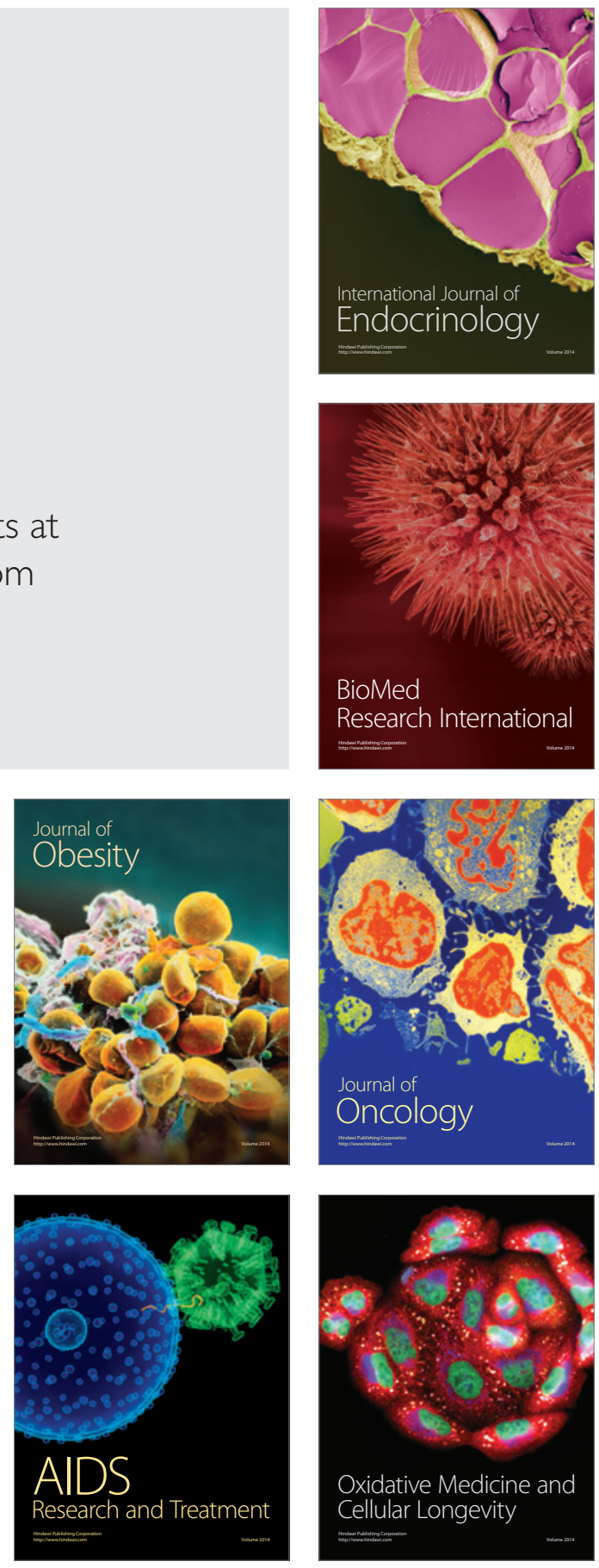